\title{
Analysis of Surgical Patient Flow at Winnipeg Health Sciences Centre
}

\author{
Y.Y. Tan ${ }^{1}$, T. Y. ElMekkawy ${ }^{1}$, Q. Peng ${ }^{1}$, B. Wright ${ }^{2}$ \\ ${ }^{1}$ Department of Mechanical and Manufacturing Engineering, University of Manitoba, \\ 15 Gilson Street, Winnipeg, MB, R3T 5V6, Canada \\ ${ }^{2}$ Winnipeg Regional Health Authority, Winnipeg, MB, Canada
}

\begin{abstract}
The Health Sciences Centre (HSC) in Winnipeg is the major trauma centre serving the entire province of Manitoba, Northwestern Ontario, and Nunavut. Therefore, it has to handle a high volume of both elective and emergent surgical patients. Because the facility always strives to provide quality care in a fast and effective manner, it initiated a research project to analyze its surgical patient flow and generate ideas on how it could be redesigned to improve the system's performance. After a year of careful analysis, all of the problems identified were grouped into six major categories, showcasing how various departments are affected by each problem. Based on this analysis, the true impact of each problem in the surgical patient flow process can be understood. Steps can now be taken to identify which problems need to be addressed, what changes should be made, and how this will benefit the entire system.
\end{abstract}

\section{Introduction}

Although the complexity of healthcare has greatly increased during the past few decades, the design of key processes has remained relatively primitive. It is so common to encounter waits, delays, and cancellations in health care that most patients and hospital staff believe that those problems are just an inescapable part of the care process. Very often, struggling hospitals have the tendency to turn to quick fixes, such as downsizing or adding more resources such as beds or equipment to address such issues. However, most of these changes have not resulted in the desired outcome $[1,2]$.

In the face of workforce shortages, an aging population, outdated hospital processes and infrastructure, increasing demand for safer care environments and better patient outcomes, remarkable operational improvements are clearly required. The average acute care area is usually overwhelmed with waits, delays, and diversions.
Recent assessments on what causes all these delays have suggested that by improving patient flow, these problems may be avoided or at least lessened. When patients, information, and materials do not timely and efficiently flow through a hospital, staff and patient satisfaction, hospital revenue, and patient safety are all negatively affected. On-time starts, turnover times, and patient flow issues affect costs and revenue, quality of care, and patient, staff, and physician satisfaction [3]. Therefore, the factors that influence the smooth and timely flow of patients through a hospital should be analysed, and methods for developing and improving patient flow should be generated.

In 2005, the Health Sciences Centre (HSC) in Winnipeg embarked on a collaborative effort with the University of Manitoba to analyze its surgical patient flow, considering the entire journey from pre to postoperative care, and develop ideas on how it could be improved through process redesign. In order to understand the HSC's patient flow, researchers spent almost a year visiting major surgical departments and carrying out observations or corresponding with staff members and patients. This paper presents the various factors that were found to be impeding smooth patient flow at the HSC.

It should be noted that this research commenced before some of the major departments that make up the HSC's patient flow, such as the operating room (OR) department, post-anaesthesia care unit (PACU), and surgical intensive care unit (SICU), moved to a new building. However, the majority of surgical patient flow processes has remained the same, and hence the results presented in this paper are still relevant.

The remainder of this paper is divided into six sections. Sections 2, 3 and 4 consist of information regarding patient flow that can be found in literature. Section 2 provides the reader a brief understanding of how poor flow is caused, Section 3 offers a simple strategy for facilitating change, and Section 4 summarizes barriers to improving patient flow. Section 5 delivers an overview of the surgical patient 
flow at HSC. In Section 6, the issues hampering patient flow at the HSC are presented. Finally, concluding remarks and an explanation of how the project will progress is given in Section 7.

\section{Literature on the Causes of Inefficient Flow}

\subsection{Bottlenecks}

Areas that are non-interchangeable resources, such as emergency departments (ED), intensive care units (ICU), OR departments and their pre and postoperative units are major bottlenecks in more healthcare facilities [2]. Bottlenecks represent a care and safety problem, as well as a cost issue. For example, if a patient is awaiting transfer from the ICU to a post-operative unit, a safety issue arises because there may be another patient who urgently requires the care that the ICU provides, and a cost is incurred because expensive ICU resources are being wasted. More often than not, these problems are not caused by a lack of effort from staff, and cannot be resolved by working harder. Rather, it is the patient flow between and among the different departments that is the source of problems.

Therefore, it must be understood that the hospital is made up of several interdependent departments, and not individual ones. The actions of one department can adversely affect another, and so patient flow throughout the entire system must be improved, rather than just in isolated departments

The key solution to bottleneck problems is to redesign the processes that create the patient flow problems. It is imperative that researchers look upstream and downstream from bottleneck units in order to make system-level changes that will solve flow issues.

For example, the Baptist Memorial Hospital strived to improve flow in its ED by beginning rapid process improvements [4]. They have now implemented the use of faxed reports between the ED and receiving units in order to eliminate time spent on holding and returning phone calls. This change, coupled with other flow improvement methods, has made vast improvements in the ED department.

\subsection{Variability}

Flow depends on the inherent variation found in the healthcare delivery system [2]. Variation is the result of clinical, flow, and professional variability. Clinical variability refers to the number of patients presenting certain clinical conditions, flow variability refers to the ebb and flow of patients arriving throughout the day, and professional variability refers to the variation in skill levels and techniques among providers.

It is commonly assumed that healthcare flow results from what appears to be random, complex and unpredictable patient needs. However, there have been studies on the effects of variability in healthcare that have challenged this belief. It has been found that the variations caused by the healthcare delivery structure, termed artificial variations, are much greater than the natural variations caused by random patient arrivals in the ED and the disease state they present [2]. It is this variation that causes avoidable problems in bottleneck areas.

Natural variation does affect flow, but it tends to become relatively constant and commonly plays a smaller role. Natural variation can be accommodated by using historical data and queuing methods to manage demand. Also, the natural variations caused by different staff competency levels and clinical abilities can also be minimized by striving to maintain staff competency and providing continual training.

In order to effectively improve patient flow, process variations much be reduced. Variability in caseloads, patient acuity, and specialty needs impacts the hospital as a whole. Although there is the notion that each patient is unique and requires their own approach, patients being treated for similar conditions are much more alike than they are different. Failure to realize this creates a culture where individual self-rule precedes over known best practices. The view that a hospital is like a workshop, where physicians are artisans that can each practice their craft in their own way, results in inconsistent and unreliable processes. In other words, personal preferences and beliefs of different clinicians introduce artificial variation in the system.

To reduce artificial variations, hospitals should have policies that do not allow such permissiveness. Caregivers must be given standard procedures that are allowed to vary according to a patient's individual needs, although these variances must serve a thoughtful purpose. As stated in [5], unwanted and random variations in processes must first be removed in order to allow a caregiver to vary intelligently from a care process.

\section{A Strategy for Improving Patient Flow}

There are many improvement policies and strategies $[2,6,7]$ that can be found in literature on patient flow. The Institute for Healthcare Improvement [6] developed the following strategy, which was employed by the project's researchers to analyze HSC's patient flow. 


\subsection{Step 1: Evaluate Flow}

Before flow improvements can be made, hospitals must first evaluate the flow of patients through their organization and determine how often patients are moved through the system in a timely, efficient manner. The frequency of parked patients (patients who are waiting for transfer from one unit to another) and hospital occupancy are good indicators of whether the hospital regularly struggles with flow problems. Parked patients demonstrate that the system is inhibiting flow, while high occupancy at midnight implies that there is little capacity for new admissions since the hospital is already full at the start of the next day. Understanding the flow process will help identify bottlenecks and uncover their root causes [7].

In order to carry out this first step, researchers visited all of the major departments that deal with surgical patients, spending time observing how things worked, in addition to interviewing numerous staff members and patients. The departments were visited in the order in which a typical elective surgical patient would actually encounter them. All of the main processes making up HSC's surgical patient flow were carefully documented, reviewed, and presented to management at the HSC in order to ensure that a full understanding had been achieved. It is the results of this step that are presented in this paper.

\subsection{Step 2: Measure and Understand Flow Variation}

Some kinds of variability cannot be eliminated, or even reduced, such as the time when patients arrive in the ED, while non-random variability can be eradicated. IHI points out that most non-random variability is driven by priorities, such as the scheduling of surgeries based on surgeons' preferences. To determine what variability exists, hospitals should look at the variation in census between and within each day of the week. Hidden, unnecessary activities divert resources, contribute to delays, and leads to overhead costs that could have been avoided. Therefore, hospitals must critically examine their operations to uncover where these occur.

The research project described in this paper has already progressed to this step. Researchers began collecting data shortly after the first step was completed, and is now in the process of analyzing all of the information. However, due to space limitations, this portion of the strategy will not be explained in this paper.

\subsection{Step 3: Test Changes to Improve Flow}

Hospitals that desire flow improvement should consider testing changes within the hospital, such as managing discharges more efficiently. In addition, facilities should also test the changes resulting from cooperation with healthcare providers outside the hospital, such as long-term care facilities and outpatient clinics. Extending the chain is a term used by the IHI to describe the process of building relationships with care providers outside the hospital. This last phase of the strategy has not yet been reached by the researchers involved in the project.

\section{Literature on the Barriers to Obtaining Smooth Flow}

Perhaps one of the major barriers to improving patient flow in hospitals is the failure to recognize flow as important. Usually, only capacity problems cause people to address the flow issue. However, many do not realize that quality problems also arise with bad flow situations [5].

In addition, the majority of hospitals are not designed to realize optimum patient flow. The organizational structure of traditional hospitals consists of silos, where no one is in charge of flow throughout the whole hospital. Although each department may achieve optimum flow for themselves, this does not translate to good flow across the hospital.

Inefficient flow itself causes additional barriers to improvements. When staff members experience a system that does not work, they lose trust in the system's efficiency and start hoarding equipment and supplies, which can cause more flow problems.

Lastly, there are the obvious political and cultural barriers. For example, optimizing the flow through operating rooms may result in scheduling changes that surgeons are dubious of whether they will really achieve improvements. Caregivers on the frontline must be given the time, information, tools, support and permission to facilitate improvements. They must be made to understand that flow issues are very real, and hold the key to improved quality care for both patients and themselves. A culture of transparency, where fixing problems is the answer as opposed to blame, must be developed. Senior leadership must eliminate barriers to flow improvement and create the will to change. 


\section{Surgical Patient Flow at the HSC}

\subsection{Patient Types}

At the Health Sciences Centre, surgical patients are either elective or emergent. Each surgical patient belongs to a particular service (e.g. neurosurgery, dental, orthopaedic), dependant on the type of surgery they require, and they are sent to the unit corresponding to the service they are under. If there are no beds available in a unit, patients are placed "off-service", meaning that they are sent to a unit that corresponds to a different service than the one they are under.

\subsubsection{Elective Patients}

For the majority of elective patients, their journey through HSC starts when they see their surgeon and surgery is agreed upon. From there, they may be requested to visit the Pre-Admission Clinic (PAC) sometime before the day of admission to the HSC. The PAC is meant to assess a patient's medical fitness for surgery and anaesthesia. It is also an opportunity for the patient, and their family, to be provided with information about what to expect before and after surgery so that they are well prepared. In addition, this clinic allows most patients to stay at home until the day of surgery instead of having to be admitted one or two days ahead, which used to be done in the past

Figure 1 depicts the flow of elective patients through the HSC. On the day of admission, patients must first stop at the Admitting department for registration before they proceed to their pre-operative unit where they are prepared for surgery. When it is time for surgery, patients are sent to the OR department and their operation is performed. After surgery, patients are transferred to the recovery room, which is usually the PACU, although they may be sent to the SICU depending on the complexity of their case. The main purpose of the recovery room is to prevent and treat complications following surgery and anaesthesia. From there, patients are finally sent to their postoperative unit where they will stay until they are fit enough to be discharged.

At the HSC, elective patients are classified as Inpatients, Same Day (SD) patients, Overnight patients, or Same Day Admission (SDA) patients. If a patient needs to be admitted a day or more preoperatively, as specified by the patient's surgeon, then that patient can only be classified as an Inpatient. The other three patient types will all be admitted on the day of surgery, and they are classified according to their expected discharge time. Patients expected to be discharged on the same day of surgery are classified as
SD patients, Overnight patients are expected to be discharged by the next day after surgery, and SDA patients are expected to be discharged only one or more days after surgery.

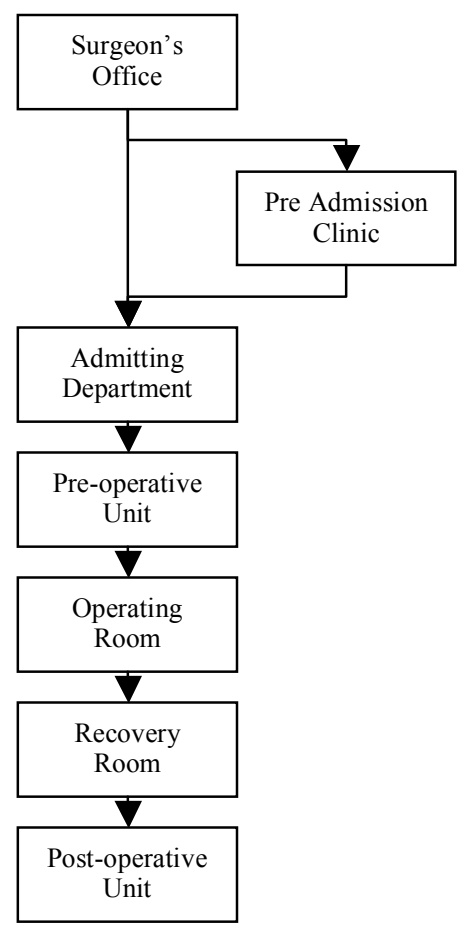

Figure 1. Path taken by most Elective Patients

\subsubsection{Emergency Patients}

The journey for the majority of emergency patients starts at the ED. From there, depending on their acuity and available OR time, patients will either be sent to a pre-operative unit before they go to the OR department, or they will go to the OR department for surgery straight away. Afterwards, they generally follow the same path as elective patients. Figure 2 illustrates the flow of emergency patients through the HSC. 


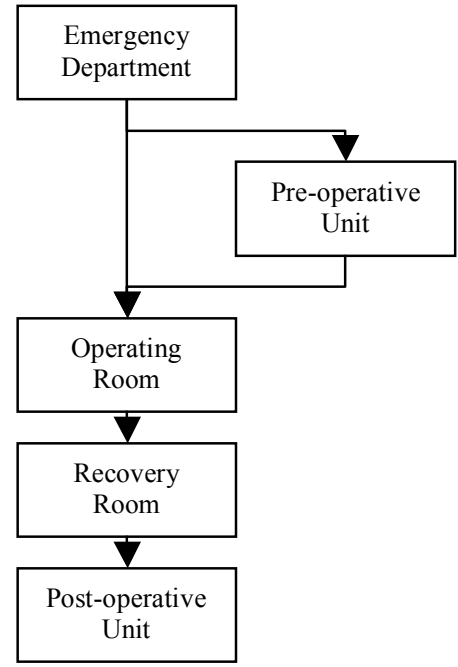

Figure 2. Path taken by most Emergency Patients

\subsection{OR Scheduling}

At the HSC, there is one slate for elective patients and one slate for emergency patients during the week. The elective slate usually schedules ten to twelve operating theatres and runs from 7:30am to 3:30pm, Monday to Friday, although there is a later start of $9 \mathrm{am}$ on Wednesdays. The emergency slate runs from $3: 30 \mathrm{pm}$ to $10: 30 \mathrm{pm}$, Monday to Friday, and usually has two staffed operating theatres. Of course very urgent emergency cases are still performed after 10:30pm.

On the weekends there is no elective slate and two operating theatres are staffed solely for emergency procedures.

\subsubsection{Elective Slate Scheduling}

In the first stage of the elective OR scheduling process at the HSC, elective OR time is allocated to the different services and a master surgical schedule is drawn up. Each block in the master surgical schedule corresponds to a particular day in a particular operating theatre, and is allocated to a specific service. It is the managers of each service who decide how to split up their blocks among the surgeons under their service. Once the blocks have been allocated to specific surgeons, the surgeons will schedule their cases into the times given to them in whatever manner they like. However, they do have to follow the rule that SD patients should be scheduled earlier in the day, before their SDA patients. This is because SDA patients may have their surgeries delayed if there are not enough post-operative beds available for them, so scheduling them later in the day will allow for more discharges in the post-operative units to be carried out before surgery begins.

\subsubsection{Emergency Slate Scheduling}

When a patient arrives in the ED, or is already inhospital, and is found to require emergency surgery, the patient's surgeon will inform the OR department. If a case is very urgent, it will be performed immediately. If this occurs during the elective slate, a few staff members from existing operating theatres, working on elective cases, will be taken out to form a new surgical team that is moved to an empty operating theatre that the emergency case can be done in. If there is no available unused operating theatre, or not enough staff members can pulled from their current elective cases, then the case will be performed in the first available operating theatre that finishes its current case, utilizing the staff originally assigned to that room. Of course this may result in the disruption of an elective case, if one is scheduled in that theatre at that time.

If a case is not very urgent, then the patient will either wait in the ED or be admitted to one of the units in the hospital until there is time for it in the emergency slate. If there is not enough emergency slate time to perform the case, it will be carried over to the next day's emergency slate, time permitting.

\section{Factors affecting Flow at the HSC}

After analysing the surgical patient flow at the HSC, all of the issues encountered were grouped into the following six key areas.

\subsection{OR Scheduling Policy}

The OR scheduling policy has a major impact on all departments in the surgical patient flow process. Because there were so many issues under this area, they were separated into five different categories.

\subsubsection{OR Time Allocations}

The first issue regarding the way cases are currently scheduled in the OR department is that the OR time allocated to elective and emergency cases are based on historical and political reasons rather than on quantifying ones. For example, OR time is not allocated for emergencies based on estimates of how much will be needed by emergency cases expected to arrive. In other words, there is no attempt to match supply with demand. When there is not enough time for emergencies, even with the emergency slate, some emergency patients will occupy beds just waiting for surgery, which subsequently affects the entire bed situation throughout the hospital. This is especially a 
problem for the less urgent cases, because they have to wait, sometimes for weeks in extreme situations, until all of the more urgent cases are taken care of, even if those cases arrived after them.

Another example is the way OR time is allocated to the different services, and subsequently, the different surgeons. The allocations do not consider waiting lists or the demand for urgent cases. When waiting lists are not taken into account, some surgeons may unfairly have a waiting list that is growing at a faster rate than others. Also, since the demand for urgent cases for each surgeon are not taken into account, the scheduling policy has no room for flexibility. When a surgeon suddenly acquires a patient who needs surgery much sooner than another patient already booked into the elective slate, he/she will often decide to replace the booked patient with the new one. Subsequently, this results in last minute changes which cause problems for many departments, which are explained in greater detail in the next section.

\subsubsection{Last Minute Changes}

Last minute changes are often made to the elective OR slate and this not only wastes the previous efforts of various departments, but it also creates extra work for them, in addition to increasing the chances of delays and cancellations.

For example, a surgeon may decide last minute to replace a patient booked into the elective slate with another one. If the surgeon decides that it would be beneficial for the patient to visit the PAC beforehand, the PAC then finds that they have to accommodate this extra patient with hardly any notice. This can lead to problems for the PAC because other patients then experience long waiting times as this add-on patient is squeezed in, and some of them may even have to have their appointments rescheduled.

In the afternoon, on the day before surgery, the preoperative units get a copy of the elective OR slate. If any last minute changes to the slate occur after this time, the pre-operative units may not be informed of them. If a patient's OR time has been changed and his/her pre-operative unit is unaware of it, the patient will arrive at a time that the unit did not expect them to and they will have to call the OR department to confirm that changes have been made.

Sometimes, patients may not be informed about changes to their OR time. If a patient's operation time was moved to a later time, the patient will arrive too early in the pre-operative unit and unnecessarily experience a longer than expected waiting time before surgery. If a patient's operation time was moved to an earlier time, he/she will simply not show up on time. When the pre-operative unit sees that the patient is late, assuming that they also know about the change, they then have to inform the OR department and Admitting so that they can call the patient. When the patient finally arrives, the pre-operative unit has to rush to get the patient prepared so that surgery can start on time. However, if a patient simply cannot make it to the hospital in time for the scheduled start, then the OR department has to find another patient to fill up the empty OR slot which further disrupts the elective slate.

Finally, the OR department is also greatly affected by changes in the OR slate because a lot of extra work is created. For example, when a surgeon replaces a patient with a more urgent one, a new case cart has to be set up and the old one dismantled. In addition, the equipment and instrumentation requirements have to re-checked, potentially leading to more changes in the elective slate in order to accommodate the case. Moreover, the nursing staff assignments must be reviewed to make sure that they are suitable for the scheduled cases.

\subsubsection{Variable Patient Volumes on the Elective Slate}

One of the major systemic causes of problems with regards to the surgical patient flow at HSC is that there is no central slating system. Surgeons book elective cases into their allocated OR time in whatever way they want as long as their resource requirements (equipment, instrumentation, operating room) are available. This means that there is no control on the overall case mix in the OR department. For example, all of the surgeons with OR time on a particular day may coincidentally decide to book all short cases which will result in a high case volume in the OR department. Other times, all of the surgeons may end up booking all long cases leading to a very low patient volume. These variable patient volumes affect both the workload and the bed situation throughout the entire hospital.

The bed situation in the hospital is directly affected by the case volume and case mix in the OR department. For example, since the PACU handles virtually all of the elective patients on the OR slate, their patient volume directly correlates with the OR slate's patient volume. Another example is that there may be a day when there are many SDA and inpatients booked on the elective slate, resulting in a large number of patients who need a bed for a lengthy period of days. This puts a strain on the bed situation and some of the newly arriving elective patients requiring those beds may have their surgery put on hold in order to wait for discharges. Sometimes, their surgery may be cancelled if no bed becomes available for them. 
The situation where a unit has reached it maximum bed occupancy always leads to problems for other units because patients start being put on hold, i.e. they cannot be moved to the unit they are meant to be going to. For example, when post-operative units are full, patients cannot be transferred to them from their recovery unit, which is usually the PACU. In turn, those patients are put on hold in the PACU, which can cause the unit to reach full capacity. Similarly, the PACU will then have to put patients on hold in the OR department. When cases in the OR department are put on hold, the surgeries of the next patients are delayed, and those patients are put on hold in their preoperative units. This chain reaction causes delays and increases the chances of the cancellations of later elective surgeries.

Like the bed situation, the workload throughout the hospital is also affected by the variability in the elective OR slate. On days with a high workload, there is a higher possibility of inadequate staffing in all units since the staffing numbers for most units do not vary from day to day. Not only does this reduce staff satisfaction and impact the quality of care, but this also increases the chances of overtime and can cause other problems.

For example, the high variability in the OR slate greatly affects the operations in the pre-operative units since it directly correlates with their patient volume. Because the staffing in those units remains constant on a daily basis, those units can be short-staffed on busy days, resulting in an increased chance of delays in getting patients to the OR department. This can then increase the chances of delays in getting operations started on time in the OR department, which can lead to overtime there, or the cancellation of cases.

Also, a higher workload means a higher pace of activities. On busy days in the OR department, there will more setting up and cleaning involved due to a higher number of turnovers. A lot of preparation is involved in getting the instrumentation and equipment ready for each case. The higher workload also means greater resource restrictions such as staff and equipment.

\subsubsection{Case Duration Estimates Not Based on Historical Times}

It is the surgeons who decide how long a case will be slated for - it is not based on their historical times. Because of this, surgeons may underestimate the amount of time that a case should be slated for, and this in turn can lead to overbooking. This may occur if surgeons think that they can do a case faster than they really can or if they forget that the case duration encompasses more than just the "cutting time" and includes set-up time and clean up. Overbooking leads to overtime for the nursing staff, causes the elective slate to run into the emergency slate time (which reduces the amount of emergency cases that can be performed), and results in cancellations.

On the other hand, some surgeons may actually overestimate the amount of time that a case should be slated for. This may be because the surgeon has experienced so many delays during his/her time at the HSC that he/she actually extends case durations to accommodate these delays.

\subsubsection{Lack of Transparency}

Actual start times are often very different from the scheduled start times on the elective slate due to a wide variety of reasons, such as inaccurate case duration estimates and delays in the surgical patient flow. Because it is hard to predict when a case will actually begin, there is no set time that an operating theatre's team (i.e. surgeon, anaesthetist, OR nurses) knows they will have to be present and ready for. It also makes it hard to estimate the number of perioperative aides, who clean the operating theatres between cases, that are required on a particular day because the finishing times of each case cannot be accurately judged by looking at the slate. Because the actual surgery times vary greatly from the slated times, it is difficult for the pre-operative units to assess patients in the order in which they will actually be sent to the OR department. Patients then experience long waiting times or have to be rushed to the OR department once they arrive in their pre-operative units.

\subsection{Pre-operative Delays}

Like many other facilities, the surgical patient flow at the HSC suffers from a variety of pre-operative delays which introduce unnecessary variability. For example, the required pre-operative information that the hospital requires before a patient's surgery, such as the patient's medical history and diagnostic test results, are often sent last minute from the surgeons' offices and may even be incomplete. This can cause problems for all of the departments that surgical patients encounter right up to the time their surgery is actually performed, such as extra calls between the departments and the surgeons' offices, or longer patient assessments in the PAC or pre-operative units. In turn, this can lead to delays in getting patients to the OR department, increasing the possibility of surgeries starting later than scheduled, which may result in overtime for the OR department's staff and perhaps cause elective case cancellations. 
Another cause of pre-operative delays is how prepared patients are for their surgery. For example, delays can occur in the PAC, pre-operative units, and the OR department, if a patient arrives at the hospital and is unable to answer important questions such as what medication they are currently taking. Another example is that patients sometimes arrive at the hospital and realize that they are scheduled for an operation that is different from the one that they thought they would be getting. In some cases, patients may change their mind and decide not to proceed with surgery, resulting in empty elective OR time that has to be filled last minute. This also means that the efforts of all the departments involved in preparing for the patient's operation (e.g. PAC, Admitting, pre-operative units, etc.) are now wasted.

\subsection{Discharge Delays}

Problems regarding the discharge of patients from their post-operative units can hamper smooth patient flow by causing delays regarding new admissions and affecting the bed situation throughout the facility. For example, a patient may be medically fit to be discharged but still waiting in the hospital because the necessary discharge order and/or medical summary are unclear or simply not done. In addition, discharging patients to certain care facilities outside the hospital can be difficult. It may be difficult to communicate with other departments that provide services, such as transport arrangements to those facilities, or the facilities may have no available beds. Also, there are no specific discharge instruction sheets for certain common procedures, which are given to patients to inform them of what to expect when they go home, so nurses must spend time filling standard ones out. Furthermore, certain units have set discharge times that need to be fulfilled in order to make room for new patients, and some patients are not notified of these times before they arrive at the hospital so they cannot make arrangements to meet them.

\subsection{Resource Management}

Like in every facility, the resources at the HSC are limited and can cause delays in every department involved in the patient flow process if not properly managed. For example, a lack of transport personnel, stretchers, or wheelchairs can cause delays in getting patients between the different departments around the hospital, a nursing shortage can lead to delays in getting patients assessed, and an assistant shortage can lead to getting rooms cleaned for arriving patients. When there is a lack of equipment or instrumentation for operations, surgery cases may have to be rescheduled in order to accommodate their requirements. Even the design of the facility and the space available creates its own challenges, such as storing supplies in an efficient manner and accommodating all of the patients, supplies, equipment, etc. that need to be there. These examples are among the many resource allocation issues that can be seen at the HSC.

\subsection{Communication}

The communication between patients and their families, nurses, surgeons, and the different departments all have the potential to negatively affect the HSC's patient flow. For example, there is no single person that patients' relatives can refer to for information about the status of a patient in-hospital, so they often find themselves having to speak to various staff members in different units, such as the Admitting department, the pre-operative units, or the recovery units. Not only is this stressful for the family, but it also takes the staff away from assessing their patients. Certain departments involved in the surgical patient flow process are sometimes left out of the information loop. For example, the Admitting department and the pre-operative units may be uninformed of changes regarding the elective OR slate, causing them unnecessary problems or extra work. Also, it can be difficult for departments to get in touch with other departments or people to seek out information or to notify them of something, such as contacting the surgeons and residents to ask if a patient can be discharged, which can delay getting certain activities done.

\subsection{The Accumulation of Delays}

With the examples on how the OR slate's patient volume affects the bed situation and workload in other departments, it is obvious that the activities in one department in the surgical patient flow process has a substantial bearing on the activities of the other departments in that same process. Most delays will not result in long periods of wasted time individually. However, it is the accumulation of delays that add up. In addition, one delay often leads to more delays which may not have happened if that first delay had never occurred. In other words, the delays found in the surgical patient flow process are often correlated.

For example, surgeons do not have to be present for a case until their patient is in the operating theatre and has been anaesthetized. Therefore, in between their cases, surgeons may leave the OR department because they will likely have to wait quite some time before their next case starts. Delays between the turnovers of 
cases, such as a lack of staff to clean up the rooms, may also prompt a surgeon to leave the OR department. If a surgeon has experienced enough delays where he/she had to wait for long amounts of time between cases, he/she will begin to anticipate all of these delays and even schedule activities between turnovers. This in turn could lead to more delays if the surgeon gets caught up elsewhere and a twenty minute trip turns into a forty-five minute event.

\section{Conclusion}

Smooth patient flow is crucial in achieving an efficiently-managed facility. At the HSC, a project was undertaken to discover how competent the processes making up their surgical patient flow was, and to generate ideas on how to improve it. After a year of observations and interviews at the HSC, it was evident that there were a variety of issues that were impeding smooth patient flow at the HSC. The major factors affecting their flow consist of the OR scheduling policy, pre-operative and discharge delays, resource management, and communication among staff and departments. Furthermore, delays that are caused by these factors are often correlated and have the ability to accumulate very quickly. Studies can now be carried out to determine which main areas need to be addressed in order to achieve optimal patient flow at the HSC.

\section{Acknowledgements}

This research project was funded by the Winnipeg Regional Health Authority and Health Science Centre. The authors gratefully thank Helga Bryant and Leanne Bernaerdt for their cooperation and support.

\section{References}

[1] M. Lambert, "Improvement and innovation in hospital operations: a key to organizational health," Frontiers of Health Services Management, vol. 20, pp. 39-45, Summer 2004.

[2] C. Haraden, and R. Resar, "Patient flow in hospitals: understanding and controlling it better," Frontiers of Health Services Management, vol. 20, pp. 3-15, Summer 2004.

[3] D. Henderson, C. Dempsey, and D. Appleby, "A case study of successful patient flow methods: St. John's Hospital," Frontiers of Health Services Management, vol. 20, pp. 25-30, Summer 2004.
[4] S.S. Horton, "Increasing capacity while improving the bottom line," Frontiers of Health Services Management, vol. 20, pp. 17-23, Summer 2004.

[5] L.P. Brideau, "Flow: why does it matter?," Frontiers of Health Services Management, vol. 20, pp. 47-50, Summer 2004.

[6] Optimizing Patient Flow: Moving Patients Smoothly Through Acute Care Settings. IHI Innovation Series white paper. Institute for Healthcare Improvement (IHI). Boston. [Online]. Available: www.IHI.org 2003.

[7] L.A. Backer, "Strategies for better patient flow and cycle time: these tried-and-true techniques will increase revenue, reduce expenses and improve satisfaction with your practice," Family Practice Management, pp. 45-50, June 2002. 